\title{
Sigma term and strangeness content of octet baryons
}

\author{
S. Dürr, ${ }^{1,2}$ Z. Fodor, ${ }^{1,2,3}$ T. Hemmert, ${ }^{4}$ C. Hoelbling, ${ }^{1}$ J. Frison, ${ }^{5}$ S. D. Katz, ${ }^{1,3}$ S. Krieg, ${ }^{1,2}$ T. Kurth, ${ }^{1}$ L. Lellouch, ${ }^{5}$ \\ T. Lippert, ${ }^{1,2}$ A. Portelli, ${ }^{5}$ A. Ramos, ${ }^{5}$ A. Schäfer, ${ }^{4}$ and K. K. Szabó ${ }^{1}$ \\ ${ }^{1}$ Bergische Universität Wuppertal, Gaussstr. 20, D-42119 Wuppertal, Germany \\ ${ }^{2}$ Jülich Supercomputing Center, Forschungszentrum Jülich, D-52425 Jülich, Germany \\ ${ }^{3}$ Institute for Theoretical Physics, Eötvös University, H-1117 Budapest, Hungary \\ ${ }^{4}$ Universität Regensburg, Universitätsstr. 31, D-93053 Regensburg, Germany \\ ${ }^{5}$ Center de Physique Théorique*, Case 907, CNRS Luminy, F-13288 Marseille, France \\ (Received 1 November 2011; published 26 January 2012)
}

\begin{abstract}
By using lattice QCD computations we determine the sigma terms and strangeness content of all octet baryons by means of an application of the Hellmann-Feynman theorem. In addition to polynomial and rational expressions for the quark-mass dependence of octet members, we use $S U(3)$ covariant baryon chiral perturbation theory to perform the extrapolation to the physical up and down quark masses. Our $N_{f}=2+1$ lattice ensembles include pion masses down to about $190 \mathrm{MeV}$ in large volumes $\left(M_{\pi} L \gtrsim 4\right)$, and three values of the lattice spacing. Our main results are the nucleon sigma term $\sigma_{\pi N}=39(4)\left({ }_{-7}^{+18}\right)$ and the strangeness content $y_{N}=0.20(7)\left({ }_{-17}^{+13}\right)$. Under the assumption of validity of covariant baryon $\chi \mathrm{PT}$ in our range of masses one finds $y_{N}=0.276(77)\left({ }_{-62}^{+90}\right)$.
\end{abstract}

DOI: 10.1103/PhysRevD.85.014509

PACS numbers: 12.38.Gc, 12.39.Fe, 14.20.Dh

\section{INTRODUCTION}

The nucleon sigma term and the nucleon strangeness content are phenomenologically important quantities that are not directly accessible to experiment. They are related to $\pi-N$ and $K-N$ scattering lengths, to the quark-mass ratio $m_{s} / m_{u d}$ (where $\left.m_{u d}=\left(m_{u}+m_{d}\right) / 2\right)$, to the hadron spectrum and even to counting rates in Higgs boson searches. They also play a key role in the direct detection of dark matter (DM): sigma terms relate the effective DM-nucleon coupling to the fundamental Lagrangian parameters that couple the DM particle to the quarks. A precise first principle determination of these quantities is thus very interesting. The sigma terms are defined as

$$
\begin{aligned}
\sigma_{\pi N} & =\left\langle N(p)\left|m_{u d}(\bar{u} u+\bar{d} d)(0)\right| N(p)\right\rangle, \\
\sigma_{\bar{s} s N} & =2\left\langle N(p)\left|m_{s} \bar{s} s(0)\right| N(p)\right\rangle .
\end{aligned}
$$

The usual way of computing $\sigma_{\pi N}$ is by using $\pi-N$ scattering data. $\sigma_{\pi N}$ cannot be directly obtained in this way, but available data can be extrapolated to the ChengDashen point, although the machinery involved in this determination is complicated. $\chi \mathrm{PT}$ can then be used to reliably extrapolate from the Cheng-Dashen point to the chiral limit [1,2].

Such an analysis was carried out in the 1980s [1,2], resulting in a value of $\sigma_{\pi N}=45 \pm 8 \mathrm{MeV}$. A later study claims to obtain a higher value [3], $\sigma_{\pi N}=64 \pm 7 \mathrm{MeV}$ which is almost 2 standard deviations away. Also it has been pointed that the uncertainties associated with these determinations are already affecting the interpretation of (direct) dark matter search experiments [4], although

*CPT is research unit UMR 6207 of the CNRS and of AixMarseille University and University Sud Toulon-Var. lattice simulations are already helping to change this situation [5].

Sigma terms of other octet members are also of phenomenological interest. Recently they have been used in the context of the hadron resonance gas model for estimating quark-mass effects in QCD thermodynamics calculations [6]. Moreover, as we will see, an analysis of all octet members can be further used to constrain the strangeness content of the nucleon.

In this paper we compute the sigma terms of the octet baryons $\sigma_{\pi X}, \sigma_{\bar{s} s X}$ for $X=N, \Lambda, \Sigma$, $\Xi$. The FeynmanHellman theorem applied to QFT relates the sigma terms to the dependence of baryon masses with respect to quark masses through:

$$
\begin{gathered}
\sigma_{\pi X}=m_{u d} \frac{\partial M_{X}}{\partial m_{u d}}, \\
\sigma_{\bar{s} s X}=2 m_{s} \frac{\partial M_{X}}{\partial m_{s}} .
\end{gathered}
$$

This opens the possibility of computing the octet sigma terms via the variation of octet masses with respect to quark masses in a lattice simulation, which is the approach that we use here. A preliminary account of this work has been given in [7]. There are other interesting quantities directly related to the previously mentioned sigma terms that are also useful for phenomenology

$$
\begin{aligned}
y_{X} & =\frac{2\langle X(p)|\bar{s} s(0)| X(p)\rangle}{\langle X(p)|(\bar{u} u+\bar{d} d)(0)| X(p)\rangle}, \\
f_{u d X} & =\frac{m_{u d}\langle X(p)|(\bar{u} u+\bar{d} d)(0)| X(p)\rangle}{M_{X}}, \\
f_{s X} & =\frac{m_{S}\langle X(p)|\bar{s} s(0)| X(p)\rangle}{M_{X}} .
\end{aligned}
$$


TABLE I. Parameters of our simulations. The errors quoted here are purely statistical. These results correspond to one of the 18 twopoint function time fit intervals that we use in our estimate of systematic uncertainties. In this particular analysis, the scales at $\beta=3.3$, 3.57, 3.7 are $a^{-1}=1616(20) \mathrm{MeV}, 2425(27) \mathrm{MeV}, 3142(37) \mathrm{MeV}$, respectively.

\begin{tabular}{|c|c|c|c|c|c|c|}
\hline$\beta$ & $a m_{u d}$ & $a m_{s}$ & $L^{3} \times T$ & Trajectories & $a M_{\pi}$ & $a M_{K}$ \\
\hline \multirow[t]{6}{*}{3.3} & -0.0960 & -0.057 & $16^{3} \times 32$ & 10000 & $0.4115(6)$ & $0.4749(6)$ \\
\hline & -0.1100 & -0.057 & $16^{3} \times 32$ & 1450 & $0.322(1)$ & $0.422(1)$ \\
\hline & -0.1200 & -0.057 & $16^{3} \times 64$ & 4500 & $0.2448(9)$ & $0.3826(6)$ \\
\hline & -0.1233 & -0.057 & $24^{3} \times 64$ & 2000 & $0.2105(8)$ & $0.3668(6)$ \\
\hline & -0.1233 & -0.057 & $32^{3} \times 64$ & 1300 & $0.211(1)$ & $0.3663(8)$ \\
\hline & -0.1265 & -0.057 & $24^{3} \times 64$ & 2100 & $0.169(1)$ & $0.3500(7)$ \\
\hline \multirow[t]{4}{*}{3.57} & -0.0318 & $0,-0.010$ & $24^{3} \times 64$ & 1650,1650 & $0.2214(7), 0.2178(5)$ & $0.2883(7), 0.2657(5)$ \\
\hline & -0.0380 & $0,-0.010$ & $24^{3} \times 64$ & 1350,1550 & $0.1837(7), 0.1778(7)$ & $0.2720(6), 0.2469(6)$ \\
\hline & -0.0440 & $0,-0.007$ & $32^{3} \times 64$ & 1000,1000 & $0.1348(7), 0.1320(7)$ & $0.2531(6), 0.2362(7)$ \\
\hline & -0.0483 & $0,-0.007$ & $48^{3} \times 64$ & 500,1000 & $0.0865(8), 0.0811(5)$ & $0.2401(8), 0.2210(5)$ \\
\hline \multirow[t]{5}{*}{3.7} & -0.007 & 0.0 & $32^{3} \times 96$ & 1100 & $0.2130(4)$ & $0.2275(4)$ \\
\hline & -0.013 & 0.0 & $32^{3} \times 96$ & 1450 & $0.1830(4)$ & $0.2123(3)$ \\
\hline & -0.020 & 0.0 & $32^{3} \times 96$ & 2050 & $0.1399(3)$ & $0.1920(3)$ \\
\hline & -0.022 & 0.0 & $32^{3} \times 96$ & 1350 & $0.1273(5)$ & $0.1882(4)$ \\
\hline & -0.025 & 0.0 & $40^{3} \times 96$ & 1450 & $0.1021(4)$ & $0.1788(4)$ \\
\hline
\end{tabular}

The first is called the strangeness content, and the other two are sometimes referred to as "dimensionless sigma terms." The latter two are directly related to the conversion of the fundamental DM-quark coupling to the effective DM-nucleon coupling.

\section{SIMULATION DETAILS AND ENSEMBLES}

The gauge and fermionic actions, as well as the algorithms used are described in [8,9]. Here it suffices to mention that we simulate QCD with two degenerate light quarks and a heavier strange quark, and that we use tree level improved Wilson fermions.

To set the lattice spacing corresponding to each value of the coupling $\beta$ and fix the quark masses to their physical values, we use $M_{\pi}, M_{K}$, and $M_{\Omega}$. We extrapolate the values of $a M_{\pi}, a M_{K}$, and $a M_{\Omega}$ to the point where the ratios $a M_{\pi} / a M_{\Omega}$ and $a M_{K} / a M_{\Omega}$ agree with the experimental values. ${ }^{1}$

As shown in Table I at $\beta=3.3$ and $\beta=3.7$ the strange quark mass is held fixed, whereas for $\beta=3.57$ we simulate at three different values of $m_{s}$ to have some lever arm to perform the small extrapolation to $m_{s}^{\text {phys }}$ (see also Fig. 1). Our data sets cover a wide range of pion masses from $M_{\pi} \sim 190 \mathrm{MeV}$ up to $M_{\pi} \sim 680 \mathrm{MeV}$, although in this analysis we only use ensembles with $M_{\pi}<550 \mathrm{MeV}$.

On every ensemble we measure the octet masses $M_{N}$, $M_{\Lambda}, M_{\Sigma}$, and $M_{\Xi}$ with valence quark masses equal to sea quark masses (only the unitary theory is considered). It is worth mentioning that these ensembles have previously been used to accurately predict the light hadron spectrum [9], including the masses of the octet baryons.

\footnotetext{
${ }^{1}$ Experimental inputs are corrected for isospin breaking and electromagnetic effects according to [9].
}

\section{CHIRAL EXTRAPOLATION}

As already stated, we simulate QCD for values of the light quark masses larger than the physical values, whereas the strange quark is close to its physical value with some lever arm to perform a small extrapolation. Thus our data requires an extrapolation in the light quark masses and an interpolation in $m_{s}$.

For this, we need to describe the quark-mass dependence of octet baryons. We will always use the tree level $S U(3)$ chiral relation to express the quark-mass dependence through the meson mass dependence via

$$
m_{u d} \propto M_{\pi}^{2}, \quad m_{s} \propto M_{\bar{s} s}^{2}=2 M_{K}^{2}-M_{\pi}^{2} .
$$

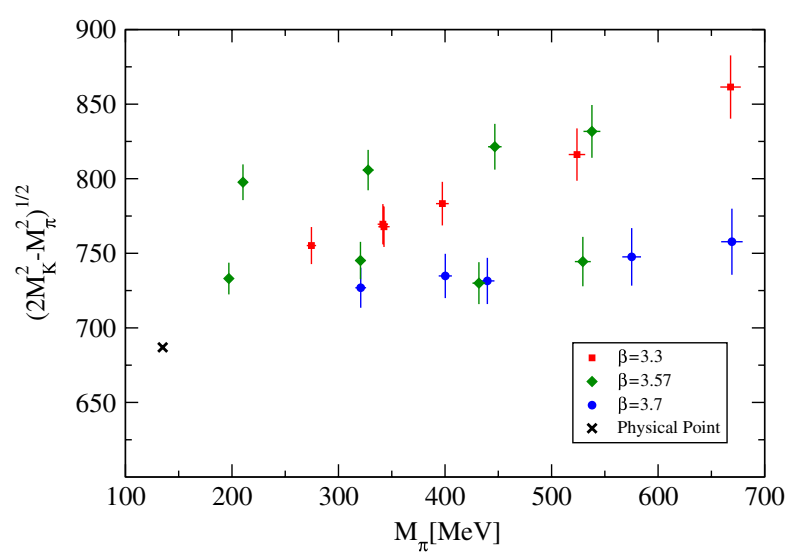

FIG. 1 (color online). Overview of our simulation points in terms of $M_{\pi}$ and $\sqrt{2 M_{K}^{2}-M_{\pi}^{2}}$. The former gives a measure of the isospin averaged up and down quark mass while the latter determines the strange quark mass. The symbols refer to the three lattice spacings, and the physical point is marked with a cross. Error bars are statistical only. 
To study this mass dependence and the associated model uncertainty, we consider two very different approaches $[9,10]$. First, we consider a regular expansion in quark masses around a non singular point where none of the quark masses vanish. For this purpose we define the expansion variables

$$
\Delta_{\pi}=\frac{M_{\pi}^{2}-\left(M_{\pi}^{\mathrm{cen}}\right)^{2}}{M_{\Omega}^{2}}, \quad \Delta_{\bar{s} s}=\frac{M_{\bar{s} s}^{2}-\left(M_{\bar{s} s}^{\mathrm{phys}}\right)^{2}}{M_{\Omega}^{2}},
$$

where $\left(M_{\pi}^{\text {cen }}\right)^{2}=\frac{1}{2}\left[\left(M_{\pi}^{\text {phys }}\right)^{2}+\left(M_{\pi}^{\text {cut }}\right)^{2}\right]$, and $M_{\pi}^{\text {cut }}$ denotes the mass of the heaviest pion included in the fit.

Note that changing the expansion point in polynomiallike formulas is simply a reshuffling of the coefficients. When interested in checking the $S U(3)$ symmetric line $m_{u d}=m_{s}$ it is far more convenient to define our expansion variables as

$$
\Delta_{\pi}=\left(\frac{M_{\pi}}{M_{\Omega}}\right)^{2}, \quad \Delta_{\bar{s} s}=\left(\frac{M_{\bar{s} s}}{M_{\Omega}}\right)^{2},
$$

where the $S U(3)$ symmetric line is characterized by $\Delta_{\pi}=$ $\Delta_{\bar{s} s}$. Because the extrapolation in $m_{s}$ is small, a linear term in $\Delta_{\bar{s} s}$ suffices.

Second, we consider a singular expansion around the $S U$ (3) chiral point $m_{u d}=m_{s}=0$, which allows for a much more constrained expansion. Low-energy processes in QCD have been extensively studied in the framework of chiral perturbation theory. The seminal work [11] and its success in explaining meson observables have pushed the study of baryons within the same framework. However, baryon $\chi \mathrm{PT}$ is far more involved. The main difficulty is that baryon masses $M_{B}$ are not small in comparison with the scale of chiral symmetry breaking $\left(\Lambda_{\chi} \sim 1 \mathrm{GeV}\right)$, and Weinberg's power counting theorem [12] fails: higherorder loop corrections contribute with powers of $M_{B} / \Lambda_{\chi}$ and are no longer small. This results in a very slow (practical) convergence of the series [13]. This convergence is partially improved by treating the baryons as heavy degrees of freedom, in what is known as the heavy baryon chiral perturbation theory [14]. Although it is reasonable for dealing with the mass dependence of baryons with light quarks, experience seems to show that observables that depend on the strange quark are not well described in this framework (see for example [15-17]). A number of possibilities have been proposed to improve the convergence and include the strange quark in the analysis. The various flavors of cutoff $\mathrm{B} \chi \mathrm{PT}$ (see [18] and references therein), or covariant $\mathrm{B} \chi \mathrm{PT}$ [19] are some examples. In this work we explore this last possibility. The interested reader can consult the review [20] and references therein.

\section{A. Regular expansions}

We have several possibilities if we decide to expand around a regular point. We can treat any octet member $X=$ $N, \Lambda, \Sigma, \Xi$ as independent and use a Taylor expansion. Using the variables defined in Eq. (5) our results are well described by the ansatz

$$
M_{X}=M_{0}^{X}+\alpha_{1}^{X} \Delta_{\pi}+\alpha_{2}^{X} \Delta_{\pi}^{2}+\beta^{X} \Delta_{\bar{s} s} .
$$

One can also use a Padé-like functional form

$$
M_{X}=\frac{M_{0}^{X}}{1-\alpha_{1}^{X} \Delta_{\pi}-\alpha_{2}^{X} \Delta_{\pi}^{2}-\beta^{X} \Delta_{\bar{s} s}} .
$$

In these expansions, $M_{0}^{X}, \alpha_{1,2}^{X}$, and $\beta^{X}$ are the 16 fitting parameters.

In principle, all octet masses should be degenerate along the $S U(3)$ symmetric line $m_{u d}=m_{s}$. We can choose to impose this constraint in our functional form. In fact our data are well fitted by the following $S U(3)$ symmetric regular expansion

$$
\begin{aligned}
M_{X}= & M_{0}+\alpha_{1}^{X} \Delta_{\pi}+\alpha_{2}^{X} \Delta_{\pi}^{2}+\left(C_{1}-\alpha_{1}^{X}\right) \Delta_{\bar{s} s} \\
& +\left(C_{2}-\alpha_{2}^{X}\right) \Delta_{\pi} \Delta_{\bar{s} s},
\end{aligned}
$$

or the corresponding Padé-like ansatz

$M_{X}=\frac{M_{0}}{1-\alpha_{1}^{X} \Delta_{\pi}-\alpha_{2}^{X} \Delta_{\pi}^{2}-\left(C_{1}-\alpha_{1}^{X}\right) \Delta_{\bar{s} s}-\left(C_{2}-\alpha_{2}^{X}\right) \Delta_{\pi} \Delta_{\bar{s} s}}$.

Now the fitting parameters have been reduced to 11: $M_{0}$, $\alpha_{1,2}^{X}$, and the coefficients $C_{1}$ and $C_{2}$ that give the dependence of octet masses along the $S U(3)$ symmetric line $m_{u d}=m_{s}$.

Note that Taylor-like and Padé-like ansatze differ in higher-order terms in $\Delta_{\pi}$ and $\Delta_{\bar{s} s}$. Thus the difference in physical results obtained by using these two functional forms measures higher-order contributions to the mass expansion.

\section{B. Covariant B $\chi$ PT}

Details of the quark-mass dependence of the octet members in $S U(3)$ baryon $\chi \mathrm{PT}$ can be found in the Appendix. Here we run the index $X=N, \Lambda, \Sigma$, $\Xi$ over octet members, and index $\alpha=\pi, K, \eta$ over the mesons.

Octet masses to next to leading order involve the nonlinear function

$$
h(x)=-\frac{x^{3}}{4 \pi^{2}}\left\{\sqrt{1-\left(\frac{x}{2}\right)^{2}} \arccos \frac{x}{2}+\frac{x}{2} \log x\right\} .
$$

The masses of the octet baryons are given by

$$
\begin{aligned}
M_{X}= & M_{0}-4 c_{\pi X} M_{\pi}^{2}-4 c_{\bar{s} s X} M_{\bar{s} s}^{2} \\
& +\sum_{\alpha=\pi, K, \eta} \frac{g_{\alpha X}}{F_{\alpha}^{2}} M_{0}^{3} h\left(\frac{M_{\alpha}}{M_{0}}\right) \\
& +4 d_{\pi} M_{\pi}^{4}+4 d_{\bar{s} s} M_{\bar{s} s}^{4},
\end{aligned}
$$

where $c_{\pi X}, c_{\bar{s} s X}$ are functions of the three low-energy constants (LEC), $b_{0}, b_{d}, b_{f}$, and the $g_{\alpha X}$ can be written in terms of the axial coupling $g_{A}$ and the ratio of couplings $\xi$ (as shown in Tables II and III). Finally $d_{\pi}, d_{\bar{s} s}$ parametrize higher-order corrections. 
TABLE II. Meson-loop couplings as a function of the $\pi N$ coupling $g_{A}$ and the quantity $\xi$. In the chiral limit $g_{A}=D+F$ and $\xi=F / D$, as described in the Appendix.

\begin{tabular}{lcccc}
\hline \hline$\alpha$ & $g_{\alpha N}$ & $g_{\alpha \Lambda}$ & $g_{\alpha \Sigma}$ & $g_{\alpha \Xi}$ \\
\hline$\pi$ & $\frac{3}{4} g_{A}^{2}$ & $\frac{1}{(1+\xi)^{2}} g_{A}^{2}$ & $\frac{\left(1+6 \xi^{2}\right)}{3(1+\xi)^{2}} g_{A}^{2}$ & $\frac{3(1-\xi)^{2}}{4(1+\xi)^{2}} g_{A}^{2}$ \\
$K$ & $\frac{\left(5-6 \xi+9 \xi^{2}\right)}{6(1+\xi)^{2}} g_{A}^{2}$ & $\frac{\left(1+9 \xi^{2}\right)}{3(1+\xi)^{2}} g_{A}^{2}$ & $\frac{\left(1+\xi^{2}\right)}{(1+\xi)^{2}} g_{A}^{2}$ & $\frac{\left(5+6 \xi \xi \xi \xi \xi^{2}\right)}{6(1+\xi)^{2}} g_{A}^{2}$ \\
$\eta$ & $\frac{(1-3 \xi)^{2}}{12(1+\xi)^{2}} g_{A}^{2}$ & $\frac{1}{3(1+\xi)^{2}} g_{A}^{2}$ & $\frac{1}{3(1+\xi)^{2}} g_{A}^{2}$ & $\frac{(1+3 \xi)^{2}}{12(1+\xi)^{2}} g_{A}^{2}$ \\
\hline \hline
\end{tabular}

TABLE III. Leading order octet quark-mass dependence, $c_{\pi X}$ and $c_{\bar{s} s X}$, as a function of the LEC $b_{0}, b_{D}, b_{F}$ (see the Appendix for more details).

\begin{tabular}{ccccc}
\hline \hline$X$ & $N$ & $\Lambda$ & $\Sigma$ & $\Xi$ \\
\hline$c_{X}^{\pi}$ & $-2\left(2 b_{0}+b_{D}+b_{F}\right)$ & $-4\left(b_{0}+b_{D} / 3\right)$ & $-4\left(b_{0}+b_{D}\right)$ & $-2\left(2 b_{0}+b_{D}-b_{F}\right)$ \\
$c_{X}^{\bar{s} s}$ & $-2\left(b_{0}+b_{D}-b_{F}\right)$ & $-2\left(b_{0}+4 b_{D} / 3\right)$ & $-2 b_{0}$ & $-2\left(b_{0}+b_{D}+b_{F}\right)$ \\
\hline \hline
\end{tabular}

To the order at which we are working only two pseudo Goldstone boson masses are linearly independent, being related through the Gell-Mann-Oakes-Rennes (GMOR) relation $3 M_{\eta}^{2}=4 M_{K}^{2}-M_{\pi}^{2}$.

When fitting lattice data to the chiral formula, it is important not to break the $S U(3)$ symmetry built into this expression. In that sense it is important that all octet masses become degenerate when $M_{\bar{s} s}=M_{\pi}$. This rules out the possibility of fixing the meson decay constants $F_{\pi, K, \eta}$ to their physical values. Here we choose to fix all of them to the common value $F_{\pi}^{\text {phys }}=92.2 \mathrm{MeV}$. This is correct to the order at which we are working. Note that we have repeated the fits including the next to leading order terms for the decay constants and obtained results compatible within statistical errors. We have also confirmed that the quality of fits decreases if a non-SU(3) symmetric ansatz is imposed by fixing each meson decay constant to its physical value.

The axial coupling $g_{A}$ is well known in phenomenology. The most precise value at the physical point is

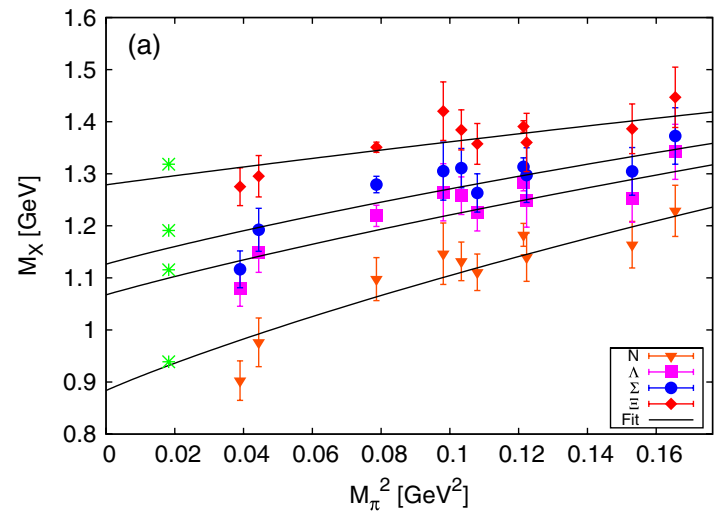

$g_{A}=1.2695(29)$ [21], and is expected to be close to the value in the chiral limit $D+F$. The ratio of couplings $\xi$ is not well determined experimentally, but there are two preferred phenomenological scenarios: $\xi=2 / 3$ and $\xi \sim 0.5$ [22].

It makes sense to try our $\chi$ PT fits both fixing $\left(g_{A}, \xi\right)=$ $(1.2695,2 / 3)$ or allowing them to be free. Regarding the higher-order contributions given by $d_{\pi}, d_{\bar{s} s}$, we can also choose to either include them in the fit, or not. In terms of how well our data are described (i.e. fit quality) the four possibilities are equally reasonable options. In Fig. 2, we show one possible $\chi$ PT fit, where we have fitted $g_{A}, \xi$ and we have not included the higher-order terms in the fit.

\section{CUTOFF, FINITE VOLUME EFFECTS, AND EXCITED-STATE CONTRIBUTIONS}

We combine our chiral extrapolation with the continuum extrapolation. It has been demonstrated in different contexts $[8,9,23,24]$ that a smeared clover action is very close to be nonperturbatively $\mathcal{O}(a)$-improved with cutoff effects

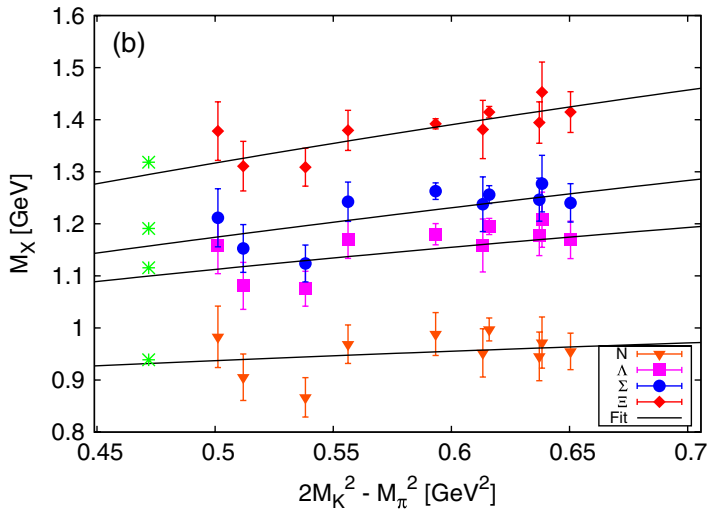

FIG. 2 (color online). Example of a $\chi$ PT fit corresponding to a particular choice of fitting interval for the correlators. In this particular case we choose to fit $g_{A}$ and $\xi$, and not to include higher-order terms. We have only used data with $M_{\pi}<410 \mathrm{MeV}$. The correlated $\chi^{2}=38.5$ for 34 degrees of freedom yields a fit quality of $\sim 0.27$. (a) Fit in the $M_{\pi}^{2}, M_{X}^{2}$ plane. Data have been corrected to the physical value of $M_{\bar{s} s}$ for a better visualization. (b) Fit in the $M_{\bar{s} s}^{2}, M_{X}^{2}$ plane. Data have been corrected to the physical value of $M_{\pi}$ for a better visualization. 

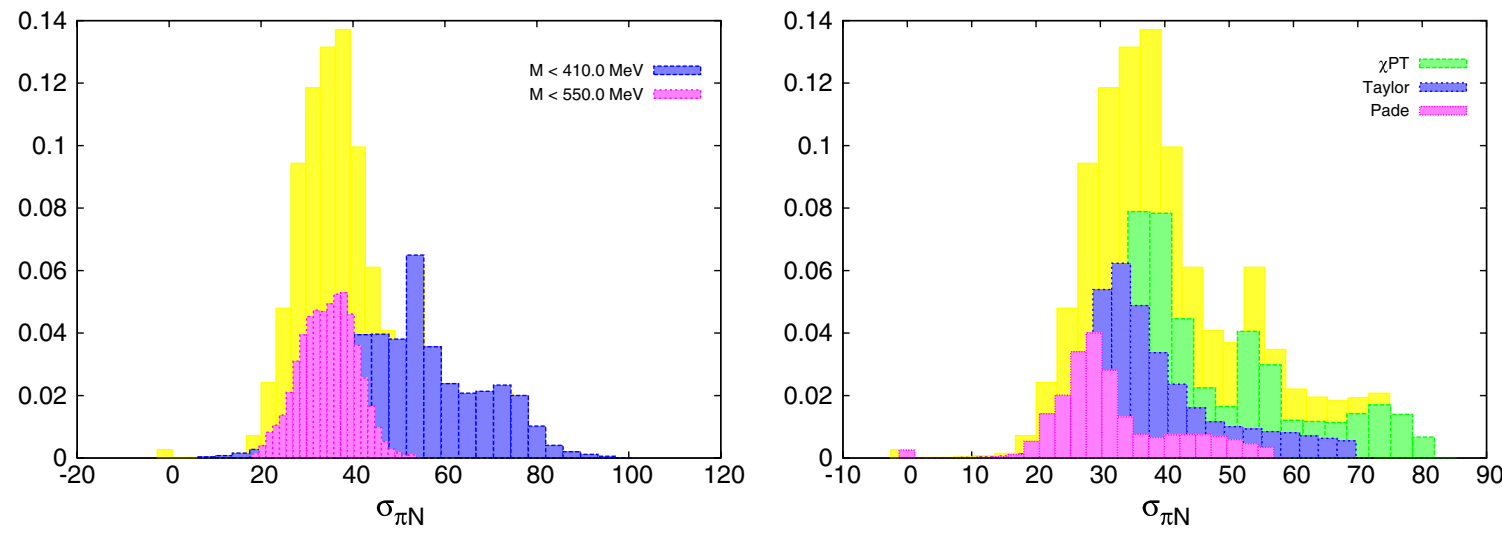

FIG. 3 (color online). Distribution of values for $\sigma_{\pi N}$ in background, and how different pion mass cuts (left panel) and functional forms (right panel) shape it.

being $\mathcal{O}\left(a^{2}\right)$. Nevertheless we cannot rule out the possibility that our results have linear discretization effects.

Guided by our experience [9] in determining light hadron masses we parametrize cutoff effects with the substitution

$$
M_{X} \rightarrow M_{X}\left(1+c_{X} a^{p}\right)
$$

with $p=1,2$. The cutoff effects are small enough in our data that they make it difficult to distinguish $a$ and $a^{2}$. In fact their coefficients are, for the most part, compatible with zero within statistical errors. Thus we will also include fits without any cutoff corrections altogether.

Stable hadron masses have leading finite-volume effects $\propto e^{-M_{\pi} L}$ [25]. In all of our ensembles the bound $M_{\pi} L \geqslant 4$ is maintained, implying negligible finite-volume effects. The detailed analysis of [9], with additional ensembles in smaller volumes, shows that finite-volume corrections are below the statistical accuracy of our data. Thus we do not add finite-volume corrections to our analysis here.

To estimate the possible contamination by excited states in the extraction of the masses from the correlators we use 18 fitting time intervals: $t_{\min } / a=5$ or 6 for $\beta=3.3$; $t_{\min } / a=7,8$, or 9 for $\beta=3.57$; and $t_{\min } / a=10,11$, or 12 for $\beta=3.7$ [9].

\section{DETERMINATION OF THE UNCERTAINTIES}

Our complete analysis includes a total of 8 formulas to extrapolate to the physical mass point: Four of them are regular expansions either imposing or not the $S U(3)$ flavor constraint, and either in the Taylor-like form or Padé-like form. The other four functional forms are derived from $S U(3)$ covariant baryon $\chi \mathrm{PT}$, where we can choose to parametrize or not higher-order contributions, and to fit $g_{A}$ and $\xi$ or fix $g_{A}$ to its physical value $g_{A}=1.2695(29)$ [21] and $\xi$ to a reasonable phenomenological value ${ }^{2} \xi=$ $2 / 3[22]$.

\footnotetext{
${ }^{2}$ We have checked that using $\xi=0.5$ (the other common phenomenological scenario) leads to very similar results.
}

To have more control over the uncertainties associated with higher-order terms in the mass extrapolation, we impose two different pion mass cuts: $M_{\pi}<410 \mathrm{MeV}$ and $M_{\pi}<550 \mathrm{MeV}$.

As explained in Sec. IV we consider three possibilities to parametrize cutoff effects which are compatible with our data: either assume that they are absent, or parametrize them as $\mathcal{O}(a)$ or $\mathcal{O}\left(a^{2}\right)$. Finite-volume corrections are not considered because they are below our statistical accuracy. Finally we repeat the full analysis with 18 different timefitting intervals for the correlators to estimate excited state contributions.

This strategy leads to $8 \times 2 \times 3 \times 18=864$ different procedures to estimate the physical value of each of the sigma terms of the octet members. The data are fitted (taking correlations into account) using all of the previously explained procedures. Each of the 864 results is weighted with the fit quality ${ }^{3}$ ( $p$-value or $Q$-value) to produce a distribution of values. The median (typical result of our analysis) is taken as our final value. The $16^{\text {th }} / 84^{\text {th }}$ percentiles (i.e. the values which, in a Gaussian distribution, correspond to $\pm 1 \sigma$ deviations from the median) yield the systematic uncertainty of our computation (see Fig. 3). This systematic uncertainty measures how different parametrizations for mass extrapolation, cutoff effects, and excited-state contributions affect the final result. The statistical uncertainty is determined by bootstrapping the whole procedure 2000 times, and computing the variance of the medians.

It is important to note here three points:

(i) Most of the previously mentioned fits are "good." Our average fit quality is 0.34 , and this does not vary significantly over our different procedures. For example fits with $M_{\pi}<410 \mathrm{MeV}$ have an average fit quality of 0.38 whereas for $M_{\pi}<550 \mathrm{MeV}$ it is 0.29

\footnotetext{
${ }^{3}$ The confidence of fit is defined as $Q=\int_{\gamma^{2}}^{\infty} d z P(z, d)$ where $P(z, d)=z^{d / 2-1} e^{-z / 2} /\left[2^{d / 2} \Gamma(d / 2)\right]$ is the probability distribution function to obtain $\chi^{2}=z$ in a fit with $d$ degrees of freedom.
} 
TABLE IV. Final results for all octet members. The first two columns give the baryon octet sigma terms [Eq. (1)], the third one the strangeness content of the octet member [Eq. (3a)], and the last two the dimensionless sigma terms [Eqs. (3b) and (3c)]. The first error is statistical and the second one is systematic.

\begin{tabular}{|c|c|c|c|c|c|}
\hline & $\sigma_{\pi X}(\mathrm{MeV})$ & $\sigma_{\bar{s} s X}(\mathrm{MeV})$ & $y_{X}$ & $f_{u d X}$ & $f_{\bar{s} s X}$ \\
\hline$N$ & $39(4)\left(\begin{array}{l}+18 \\
-7\end{array}\right)$ & $67(27)\left(\begin{array}{l}+55 \\
-47\end{array}\right)$ & $0.20(7)\left(\begin{array}{l}+13 \\
-17\end{array}\right)$ & $0.042(5)\left({ }_{-4}^{+21}\right)$ & $0.036(14)\left(\begin{array}{l}+30 \\
-25\end{array}\right)$ \\
\hline$\Lambda$ & $29(3)\left(\begin{array}{c}+11 \\
-5\end{array}\right)$ & $180(26)\left(\begin{array}{c}+48 \\
-77\end{array}\right)$ & $0.51(15)\left({ }_{-27}^{+48}\right)$ & $0.027(3)\left({ }_{-10}^{+5}\right)$ & $0.083(12)\left(\begin{array}{c}+23 \\
-31\end{array}\right)$ \\
\hline$\Sigma$ & $23(3)\left({ }_{-3}^{+19}\right)$ & $245(29)\left({ }_{-72}^{+50}\right)$ & $0.82(21)\left(\left(_{-39}^{+87}\right)\right.$ & $0.019(3)\left({ }_{-3}^{+17}\right)$ & $0.104(12)\left(\begin{array}{l}+23 \\
-31\end{array}\right)$ \\
\hline$\Xi$ & $16(2)\left(\begin{array}{c}+8 \\
-3\end{array}\right)$ & $312(32)\left(\begin{array}{c}+72 \\
-77\end{array}\right)$ & $1.7(5)\left(\begin{array}{l}+1.9 \\
-0.7\end{array}\right)$ & $0.0116(18)\left({ }_{-22}^{+59}\right)$ & $0.120(13)\left({ }_{-30}^{+30}\right)$ \\
\hline
\end{tabular}

[see Fig. 3(a)]. For covariant B $\chi \mathrm{PT}$, Taylor and Padé functional forms the $p$-values are, respectively, 0.30 , 0.47 , and 0.29 [see Fig. 3(b)]. The fit quality is almost insensitive to how we choose to parametrize the cutoff effects, or what time intervals we use to fit correlators. Although there is some variation, we cannot rule out any of our fits based on how well our data are described.

(ii) Adding more variations does not increase the systematic uncertainty. For example adding a pion mass cut of $M_{\pi}<680 \mathrm{MeV}$ (i.e. including all our ensembles in the fit), the covariant $\mathrm{B} \chi \mathrm{PT}$ functional form gives fit qualities of $2 \times 10^{-6}$. Even if they are included in our analysis, these fits do not contribute to our distribution of values or to our final results and estimates of systematic uncertainties.

(iii) On the other hand, eliminating some of the analysis typically results in a compatible final value but with a smaller systematic uncertainty. For example had we chosen to perform our analysis only with $M_{\pi}<$ $550 \mathrm{MeV}$ the systematic uncertainty of $\sigma_{\pi N}$ would have dropped by almost 50\%. [see Fig. 3(a)].

\section{RESULTS}

Following the method described in the previous section, we arrive at the results quoted in Table IV.

For the nucleon sigma term our result agrees with the "canonical" determination coming from $\pi-N$ scattering data [2] of $\sigma_{\pi N}=45 \pm 8 \mathrm{MeV}$, but with a larger uncertainty, and disfavors the larger value $\sigma_{\pi N}=64 \pm 7 \mathrm{MeV}$ from [3]. It also agrees with recent estimates that use lattice data [15,26-32] though these assume a particular functional form for the light-quark-mass dependence while we consider a whole range of forms. Another study considers a variety of chiral forms, but with pion masses only down to $300 \mathrm{MeV}$ [33], yielding values in the range 42 to $84 \mathrm{MeV}$, with a statistical accuracy of about $15 \mathrm{MeV}$. Our value is less consistent with the determinations of [34,35] that are close to the higher value (of [3]) for the sigma term.

We believe that the agreement within error of our results obtained from extrapolations using very different functional forms enhances the credibility of our final results.

In Table $\mathrm{V}$ we give the contribution of each source of systematic uncertainty to the final error. Although we show the results for $\sigma_{\pi N}$, the conclusion that the chiral extrapolation dominates the systematic uncertainty of our computation is generally true.

To reduce this source of systematic uncertainty we would need to add ensembles at lower pion masses. From the value of the uncertainties of $\sigma_{\pi N}$ at $M_{\pi}=200 \mathrm{MeV}$, we can roughly guess the gain that would be obtained by having equally precise data at the physical point. Such data would imply a reduction of around 50\% in the systematic error. On the other hand the situation for both $\sigma_{\bar{s} s N}$ and $y_{N}$ is less clear. Our data set has a much smaller range of strange quark masses than light quark masses. If we add the fact that the contribution of the strange quark to the nucleon mass seems to be small "per se", it is natural that the final results for both $\sigma_{\bar{s} s N}$ and $y_{N}$ to show large statistical and systematic uncertainties. Only by adding simulation points for a wider range of strange quark masses one can increase the chances of getting a precise determination of these quantities without making an additional assumption which relates $m_{s}$ to $m_{u d}$ dependence as in $S U(3)$ baryon $\chi \mathrm{PT}$.

Regarding the other octet members, the values of the sigma terms agrees with other determinations based on lattice data and a low-energy effective field theory approach $[30,31]$.

\section{$\chi$ PT consistency check}

Our objective in this paper is to determine the sigmaterms and strangeness contents of the octet baryons at the physical point. To this end, we generated ensembles close to the physical value of the strange quark mass and as close as possible to the physical light quark mass, with a large enough lever arm in the light sector to perform a credible extrapolation to the physical point.

TABLE V. Contribution to the total uncertainty of the different sources of systematic error.

\begin{tabular}{lc}
\hline Source of systematic error & Error on $\sigma_{\pi N}(\mathrm{MeV})$ \\
\hline Chiral extrapolation: & \\
Pion mass range & 9.0 \\
Functional form & 5.5 \\
Continuum extrapolation & 1.9 \\
\hline \hline
\end{tabular}


TABLE VI. Values of the LECs obtained from our $\chi \mathrm{PT}$ fits. Total errors (statistics and systematics included) are computed in the same way as for the sigma terms. For the reasons noted in the text, we do not consider these errors to be reliable.

\begin{tabular}{lccccc}
\hline \hline$M_{0}(\mathrm{GeV})$ & $b_{0}\left[\mathrm{GeV}^{-1}\right]$ & $b_{D}\left[\mathrm{GeV}^{-1}\right]$ & $b_{F}\left[\mathrm{GeV}^{-1}\right]$ & $g_{A}$ & $\xi$ \\
\hline $0.75(15)$ & $-0.71(24)$ & $0.103(60)$ & $-0.359(72)$ & $0.92(13)$ & $0.402(90)$ \\
\hline \hline
\end{tabular}

Such a set of ensembles, however, is not ideally suited to determine the $S U(3) \chi$ PT LECs, which are defined in the chiral limit. That would require simulations with a strange quark mass approaching the chiral limit. This means that the LECs extracted from our fits have uncertainties which we cannot properly control. Nevertheless, it is worth noting that the values obtained are roughly consistent with general expectations from phenomenology [21] and other lattice studies [30,31] (see Table VI). This further enhances the credibility of the results obtained at the physical point.

\section{CONCLUSIONS}

We computed the sigma terms of light octet baryons by studying their mass dependence on quark masses through the Feynman-Hellman theorem. We also obtained the strangeness content and dimensionless sigma terms for all octet members. We estimated the systematic uncertainties associated with the choice of parametrization for this mass dependence by considering two very different expansion schemes. We considered regular expansions of octet masses, and a singular three-flavor, low-energy effective field theory approach. Within each of the approaches we estimated the impact of higher-order contributions. Moreover we varied the range of fitted quark masses, cutoff parametrizations and excited-state contributions.

The most interesting quantities are the ones associated with the nucleon. Our final value for $\sigma_{\pi N}=39(4)\left({ }_{-7}^{+18}\right)$ favors the "low scenario" with $\sigma_{\pi N}=45 \pm 8 \mathrm{MeV}$ of $[1,2]$, but the size of our uncertainties does not allow us to exclude the higher value of [3]. Regarding the strangeness content we obtained a value with large statistical and systematic uncertainties $y_{N}=0.20(7)\left({ }_{-17}^{+13}\right)$. The situation is a bit better (thanks to correlations between sigma terms and the extrapolated value of the mass) for the case of the dimensionless sigma terms $f_{u d N}=0.021(3)\left({ }_{-4}^{+11}\right)$ and $f_{\bar{s} s N}=0.072(29)\left({ }_{-50}^{+60}\right)$, that are the quantities of interest for direct DM searches.

This work shows the difficulties associated with obtaining precise values for these form factors in a model independent way. Even with pion masses as low as $190 \mathrm{MeV}$ the mass extrapolation dominates the systematic uncertainties of our final results. This explicitly shows the risks of using lattice data and only one approach to perform the mass extrapolation. A proper estimation of systematic uncertainties should include the uncertainty associated with the model that is used, and, at least in our case, this is one of the main sources of systematic uncertainty.
For $\sigma_{\pi N}$ the only way to increase the precision of our computation without assuming a model would be to generate data at lower quark masses. Replacing the extrapolation by an interpolation drastically reduces the uncertainty in the final result, as we have explicitly seen by considering the values of the sigma terms at $M_{\pi}=200 \mathrm{MeV}$.

For the case of the strangeness content of the nucleon and $\sigma_{\bar{s} s N}$, the situation is even more delicate. First, $N_{f}=$ $2+1$ lattice ensembles usually bracket the physical value of $m_{s}$ with a small lever arm. This is more than sufficient to determine physical observables, but it is not the ideal setup to estimate how physical quantities change with $m_{s}$. Second, the contribution of the strange quark to the nucleon mass seem to be small per se. In principle, the first problem can be solved by measuring the nucleon mass for a larger range of strange quark masses. The second problem is solved if we restrict our analysis to three-flavor $\mathrm{B} \chi \mathrm{PT}$. This low-energy effective field theory approach relates the $m_{u d}$ dependence of octet members to its $m_{s}$ dependence, and thus constrains $y_{N}$. The value of the nucleon strangeness content obtained from the covariant $\mathrm{B} \chi \mathrm{PT}$ analysis alone has a small relative error $y_{N}=0.276(77)\left({ }_{-62}^{+90}\right)$, illustrating the success of the idea. However, since we do not have a large enough range of strange quark masses to establish the validity of $S U(3), \mathrm{B} \chi \mathrm{PT}$, this result must be considered model dependent. Note also that a direct calculation of the corresponding disconnected contribution suggests a very small $y_{N}$ value, barely compatible with the $\mathrm{B} \chi \mathrm{PT}$ result [36].

\section{ACKNOWLEDGMENTS}

Computations were performed using HPC resources from F.Z. Jülich and from GENCI-[IDRIS/ CCRT] (Grant No. 52275) and clusters at Wuppertal and CPT. This work is supported in part by EU Grants No. I3HP, FP7/2007-2013/ERC, No. 208740, No. MRTN-CT-2006035482 (FLAVIAnet), DFG Grants No. FO 502/2, SFB-TR 55, by CNRS Grant GDR No. 2921, and PICS No. 4707. The authors also thank Martin Jung for pointing out an error in the normalization of the dimensionless sigma terms.

\section{APPENDIX: SKETCH OF THREE-FLAVOR COVARIANT BARYON $\chi$ PT}

In this appendix, we give a brief outline of the derivation of our chiral fit form (12). Working to $\mathcal{O}\left(p^{3}\right)$, we use the standard octet baryon Lagrangians [37,38] 


$$
\begin{aligned}
\mathcal{L}_{M B}^{(1)}= & \operatorname{Tr}\left(\mathrm{i} \bar{B} \gamma^{\mu} D_{\mu} B-M_{0} \bar{B} B+\frac{D}{2} \bar{B} \gamma^{\mu} \gamma_{5}\left\{u_{\mu}, B\right\}\right. \\
+ & \left.\frac{F}{2} \bar{B} \gamma^{\mu} \gamma_{5}\left[u_{\mu}, B\right]\right), \\
\mathcal{L}_{M B}^{(2)}= & \operatorname{Tr}\left(b_{D} \bar{B}\left\{\chi_{+}, B\right\}+b_{F} \bar{B}\left[\chi_{+}, B\right]+\ldots\right) \\
& +b_{0} \operatorname{Tr}(\bar{B} B) \operatorname{Tr}\left(\chi_{+}\right)+\ldots,
\end{aligned}
$$

with the chiral tensors

$$
\begin{gathered}
D_{\mu} B=\partial_{\mu} B+\left[\Gamma_{\mu}, B\right], \\
\Gamma_{\mu}=\frac{1}{2}\left(u^{\dagger}\left(\partial_{\mu}+\ldots\right) u+u\left(\partial_{\mu}+\ldots\right) u^{\dagger}\right), \\
u_{\mu}=\mathrm{i}\left(u^{\dagger}\left(\partial_{\mu}+\ldots\right) u-u\left(\partial_{\mu}+\ldots\right) u^{\dagger}\right), \\
\chi_{ \pm}=u^{\dagger} \chi u^{\dagger} \pm u \chi^{\dagger} u, \\
\chi=2 B_{0}(M+\ldots),
\end{gathered}
$$

where $M$ denotes the (diagonal) quark-mass matrix. To the order we are working, there is no contribution from the $\mathcal{O}\left(p^{3}\right)$ Lagrangian, $\mathcal{L}_{M B}^{(3)}$, to the quark-mass dependence of the octet baryon masses. The coupling constants $D, F$ of Eq. (A1) are related to the actual couplings of the nucleon in the chiral limit through $D+F=\lim _{u, d, s \rightarrow 0} g_{A}$ and $F / D=\lim _{u, d, s \rightarrow 0} \xi$, whereas $M_{0}$ corresponds to the chiral limit value of the mass of the baryon octet. The field $u(x)=\sqrt{U(x)}$ describes a nonlinear matrix representation of the (quasi-) Goldstone boson fields and $B$ is the matrixvalued interpolating field for a spin $1 / 2$ octet baryon. The next-to-leading order low-energy constants $b_{D}, b_{F}, b_{0}$ govern the leading quark-mass contributions to the mass of a spin $1 / 2$ octet baryon, with $\mathcal{M}$ denoting the quarkmass matrix for three light flavors $u, d$, and $s$. Furthermore, we note that the parameter $B_{0}$ is a measure of the size of the chiral condensate $\langle 0|\bar{q} q| 0\rangle$ (in the chiral limit) [39]

$$
B_{0} \equiv-\frac{\langle 0|\bar{q} q| 0\rangle_{m_{q} \rightarrow 0}}{F_{0}^{2}}
$$

where the low-energy constant $F_{0}$ is identified with the value of the octet Goldstone boson decay constant in the chiral limit. Finally, for the calculation at hand one needs to know the chiral meson Lagrangian up to $\mathcal{O}\left(p^{2}\right)$ [39]

$$
\begin{aligned}
\mathcal{L}_{M}^{(2)}= & \frac{F_{0}^{2}}{4} \operatorname{Tr}\left\{\left(\partial_{\mu}+\ldots\right) U^{\dagger}\left(\partial^{\mu}+\ldots\right) U\right. \\
& \left.+\chi^{\dagger} U+\chi U^{\dagger}\right\} .
\end{aligned}
$$

Generalizing the SU(2) calculation of [27] along the lines of [31], we obtain the leading one-loop result for the mass of a nucleon to $\mathcal{O}\left(p^{3}\right)$

$$
\begin{aligned}
M_{N}= & M_{0}-4\left(b_{0}+\left(b_{D}+b_{F}\right) / 2\right) \bar{M}_{\pi}^{2} \\
& -2\left(b_{0}+b_{D}-b_{F}\right) \bar{M}_{\bar{s} s}^{2}-\sum_{\alpha=\pi, K, \eta} \frac{g_{\alpha N} \bar{M}_{\alpha}^{3}}{4 \pi^{2} F_{\alpha}^{2}} \\
& \times\left\{\sqrt{1-\frac{\bar{M}_{\alpha}^{2}}{4 M_{0}^{2}}} \arccos \frac{\bar{M}_{\alpha}}{2 M_{0}}+\frac{\bar{M}_{\alpha}}{4 M_{0}} \log \frac{\bar{M}_{\alpha}^{2}}{M_{0}^{2}}\right\}+\mathcal{O}\left(p^{4}\right) .
\end{aligned}
$$

To the chiral order we are working here, the meson-loop couplings $g_{\pi N}, g_{K N}, g_{\eta N}$ can be expressed in terms of the two SU(3) parameters $F$ and $D$, as given in Table VII. For generality, we have expressed the Goldstone boson decay constant $F_{0}$ entering the loop contributions via three different symbols $F_{\alpha}$ to account for the individual contribution of the pion, kaon, and eta-cloud of the nucleon.

For a fit to lattice QCD data, it is convenient to slightly rewrite (A9) without changing the expression at the order at which we are working:

(1) We express the GMOR-masses for $\bar{M}_{K}, \bar{M}_{\eta}$ in (A10) as a function of the GMOR mass of the pion and of the mass-parameter $M_{\bar{s} s}$ introduced in (4):

$$
\begin{gathered}
\bar{M}_{K}^{2} \rightarrow \frac{1}{2}\left(\bar{M}_{\pi}^{2}+M_{\bar{s} s}^{2}\right), \\
\bar{M}_{\eta}^{2} \rightarrow \frac{1}{3}\left(\bar{M}_{\pi}^{2}+2 M_{\bar{s} s}^{2}\right),
\end{gathered}
$$

which amounts to a change of variables.

(2) We identify the GMOR-mass $\bar{M}_{\pi}$ of (4) with the corresponding lowest-lying $0^{-}$-boson mass $M_{\pi}$ in each simulation. Possible deviations in the quarkmass dependence from the linear GMOR behavior predicted in (4) can only affect $\mathcal{O}\left(p^{4}\right)$ corrections (A10).

(3) The Goldstone boson decay constants $F_{\alpha}$ in (A10) are identified with the decay constant $F_{\pi}^{\text {phys }}$. Other assignments compatible with SU(3) symmetry might be chosen as discussed in Sec. III B.

TABLE VII. Summary of meson-loop couplings $g_{\alpha B}$ in terms of the low-energy constants $D, F$.

\begin{tabular}{lcccc}
\hline \hline$g_{\alpha B}$ & $N$ & $\Lambda$ & $\Sigma$ & $\Xi$ \\
\hline$\pi$ & $\frac{3}{4}(D+F)^{2}$ & $D^{2}$ & $\frac{1}{3}\left(D^{2}+6 F^{2}\right)$ & $\frac{3}{4}(D-F)^{2}$ \\
$K$ & $\frac{1}{6}\left(5 D^{2}-6 D F+9 F^{2}\right)$ & $\frac{1}{3}\left(D^{2}+9 F^{2}\right)$ & $\left(D^{2}+F^{2}\right)$ & $\frac{1}{6}\left(5 D^{2}+6 D F+9 F^{2}\right)$ \\
$\eta$ & $\frac{1}{12}(D-3 F)^{2}$ & $\frac{1}{3} D^{2}$ & $\frac{1}{3} D^{2}$ & $\frac{1}{12}(D+3 F)^{2}$ \\
\hline \hline
\end{tabular}


(4) With the same reasoning as in the previous item, at chiral order $\mathcal{O}\left(p^{3}\right)$ we can identify the chiral limit couplings $\tilde{g}_{\pi N}, \tilde{g}_{K N}, \tilde{g}_{\eta N}$ with the physical couplings $g_{\pi N}, g_{K N}, g_{\eta N}$, resulting in Table II. The unknown ratio $\xi$ of couplings is either treated as an external input to our fits or is left as a free fit parameter.

With a very similar derivation for the case of the octet hyperons, we finally arrive at our fit function (12).
[1] R. Koch, Z. Phys. C 15, 161 (1982).

[2] J. Gasser, H. Leutwyler, and M. Sainio, Phys. Lett. B 253, 252 (1991)

[3] M. Pavan, I. Strakovsky, R. Workman, and R. Arndt, PiN Newslett. 16, 110 (2002).

[4] J. R. Ellis, K. A. Olive, and C. Savage, Phys. Rev. D 77, 065026 (2008).

[5] J. Giedt, A. W. Thomas, and R. D. Young, Phys. Rev. Lett. 103, 201802 (2009).

[6] S. Borsanyi et al. (Wuppertal-Budapest Collaboration), J. High Energy Phys. 09 (2010) 073

[7] S. Durr, Z. Fodor, J. Frison, T. Hemmert, C. Hoelbling et al., Proc. Sci., LATTICE2010 (2010) 102.

[8] S. Durr et al., Phys. Rev. D 79, 014501 (2009).

[9] S. Durr et al., Science 322, 1224 (2008).

[10] L. Lellouch, Proc. Sci., LATTICE2008 (2008) 015.

[11] J. Gasser and H. Leutwyler, Ann. Phys. (N.Y.) 158, 142 (1984).

[12] S. Weinberg, Physica A (Amsterdam) A96, 327 (1979).

[13] J. Gasser, M. Sainio, and A. Svarc, Nucl. Phys. B307, 779 (1988).

[14] E. E. Jenkins and A. V. Manohar, Phys. Lett. B 255, 558 (1991).

[15] B. Borasoy and U.-G. Meissner, Ann. Phys. (N.Y.) 254, 192 (1997)

[16] J. F. Donoghue, B. R. Holstein, and B. Borasoy, Phys. Rev. D 59, 036002 (1999).

[17] B. Borasoy, Eur. Phys. J. C 8, 121 (1999).

[18] R. D. Young, D. B. Leinweber, and A. W. Thomas, Prog. Part. Nucl. Phys. 50, 399 (2003).

[19] M. Dorati, T. A. Gail, and T. R. Hemmert, Nucl. Phys. A798, 96 (2008).

[20] V. Bernard, Prog. Part. Nucl. Phys. 60, 82 (2008).
[21] K. Nakamura et al. (Particle Data Group Collaboration), J. Phys. G 37, 075021 (2010).

[22] T. Cheng and L.-F. Li, Phys. Rev. D 57, 344 (1998).

[23] S. Capitani, S. Durr, and C. Hoelbling, J. High Energy Phys. 11 (2006)028.

[24] R. Hoffmann, A. Hasenfratz, and S. Schaefer, Proc. Sci., LAT2007 (2007) 104.

[25] M. Luscher, Commun. Math. Phys. 104, 177 (1986).

[26] D. B. Leinweber, A. W. Thomas, and R. D. Young, Phys. Rev. Lett. 92, 242002 (2004).

[27] M. Procura, T. R. Hemmert, and W. Weise, Phys. Rev. D 69, 034505 (2004).

[28] M. Procura, B. Musch, T. Wollenweber, T. Hemmert, and W. Weise, Phys. Rev. D 73, 114510 (2006).

[29] H. Ohki, H. Fukaya, S. Hashimoto, T. Kaneko, H. Matsufuru et al., Phys. Rev. D 78, 054502 (2008).

[30] R. Young and A. Thomas, Phys. Rev. D 81, 014503 (2010).

[31] J. Martin Camalich, L. Geng, and M. Vicente Vacas, Phys. Rev. D 82, 074504 (2010).

[32] R. Horsley, talk at Lattice 2011.

[33] A. Walker-Loud, H.-W. Lin, D. Richards, R. Edwards, M. Engelhardt et al., Phys. Rev. D 79, 054502 (2009).

[34] C. Alexandrou et al. (European Twisted Mass Collaboration), Phys. Rev. D78, 014509 (2008).

[35] K.-I. Ishikawa et al. (PACS-CS Collaboration), Phys. Rev. D 80, 054502 (2009).

[36] A. Schäfer, talk at Lattice 2011.

[37] J. A. Oller, M. Verbeni, and J. Prades, J. High Energy Phys. 09 (2006) 079.

[38] M. Frink and U.-G. Meissner, Eur. Phys. J. A 29, 255 (2006).

[39] J. Gasser and H. Leutwyler, Nucl. Phys. B250, 517 (1985). 